\title{
STEAM Apps: Weaving numerical and geometric comprehension with the fingers
}

\author{
Keywords \\ Digital Technology, STEAM Education, Enaction, Ecological psychology, Ethnomathematics.
}

STEM and STEAM education promote integration between science, technology, engineering, math, and the arts. The latter aims to promote deep and collaborative learning of students, through the integration of the curriculum in $\mathrm{K}-12$ science education. STEAM promotes the embodiment of cognition through learning by doing. The enactive and ecological approaches to cognition presented auspicious evidence for the role of incarnation and gesture in learning with digital technology. This work aims to provide design and concept evidence on the scope of enactive and ecological approaches within the framework of STEAM learning with digital and emerging technologies focused on the concept of tissue. We place ourselves epistemologically from the embodied design for the development of educational technology centered on "learning is moving in some way" following Dor Abrahamson who reveals the enactive and ecological approaches to learning science. Also, we are framed in Claudio Aguayo's UX and UI user experience designs with emerging technologies. In the case of digital technologies, we pay special attention to learning mathematics through the knowledge of numbers and operations with the fingers. To do this, we present a prototype of Apps inspired by Nathalie Sinclair's Touchcounts and Touchtimes, with variations in haptic perception for the emergence of numbers and operations through touching and tracing movements with the fingers on the touch screen. Regarding emerging technologies, we rely on augmented reality and virtual reality. In the case of augmented reality, we focus on learning science and geometry through the spider web. The idea is that students can see the different planes of a spider web and identify different regular and irregular geometric figures, as well as explore the architecture of the web and its properties. In relation to virtual reality, we focus on Mapuche textile art (indigenous people of southern Chile) where we explore the characteristic fabrics of the culture within the "ruca" (characteristic home of the Mapuches). First, students design Mapuche textile fabrics from geometric patterns, applying symmetry and fractals. Then with virtual reality glasses, they can navigate inside the ruca to learn details of its architecture and the fabrics most used by the Mapuche culture. Our STEAM approach to app development consists of an integrated learning ecosystem that enhances digital and immersive experiences for learning about math, science, and art using engineering and technology. Finally, we can conclude that our approach describes education as a process of cognitive assembly embodied in perception and guided action with different types of artifacts through digital affordances. We highlight the role of the disciplinary integration of Science, Technology, Engineering, Arts and Mathematics to understand and strategically reveal the scientific cultural potential of our native people "Los Mapuches". 